\title{
UN MODELO DE AUTOAPRENDIZAJE CON INTEGRACIÓN DE LAS TIC Y LOS MÉTODOS DE GESTIÓN DEL CONOCIMIENTO
}

\author{
(AN AUTONOMOUS MODEL OF LEARNING WITH INTEGRATION OF THE TIC AND THE \\ METHODS OF ADMINISTRATION OF THE KNOWLEDGE)
}

\author{
Raúl Rubén Fernández Aedo \\ Elme Carballos Ramos \\ Universidad de Ciego de Ávila (Cuba) \\ Martín E. Delavaut Romero \\ Universidad de Comaguen (Argentina)
}

\section{RESUMEN}

Las TIC ofrecen un nuevo reto al sistema educativo: pasar de un modelo unidireccional de formación, donde por lo general los saberes recaen en el profesor o en su libro de texto, a modelos más abiertos y flexibles, donde la información situada en grandes bases de datos tiende a ser compartida entre diversos alumnos. Por otra parte, se rompe la exigencia de que el profesor esté presente en el aula y tenga bajo su responsabilidad a un único grupo de alumnos. El alumno puede interactuar con otros compañeros y profesores que no tienen porqué estar situados en su misma aula. El modelo educativo que se base en recursos requiere de un cambio de paradigma para los educadores. Nuevas y variadas herramientas son una parte central del proceso de aprendizaje, y no sólo un apoyo a la enseñanza tradicional.

Palabras clave: tecnologías de la información y el conocimiento (TIC), modelo educativo, herramientas, aprendizaje.

\begin{abstract}
The TIC offers a new challenge to the educational system: to pass of an unidirectional model of formation, where in general the knowledge relapse in the professor or in their text book, to more open and more flexible models, where the information located in big databases spreads to be shared among diverse students. On the other hand, it breaks the demand that the professor is present in the classroom and have under his responsibility to an only group of students. The student can exchange with other partners and professors that don't have reason to be located in his same classroom. The educational
\end{abstract}


pattern that is based on resources requires of a paradigm change for the educators. New and varied tools are a central part of the learning process, and not only a support to the traditional teaching.

Keywords: technologies of the information and the knowledge (TIC), model educational, tools, learning.

Un ambiente de aprendizaje que se sustente en el modelo educativo basado en recursos entrega variadas oportunidades y beneficios a los alumnos y profesores, además de cambios en los roles. De acuerdo con Fernández (2007) bajo este enfoque los alumnos deben ser capaces de planificar la búsqueda, localizar, recuperar, procesar, registrar, presentar y evaluar información, frente a lo cual los profesores deben estimular a sus alumnos para:

- $\quad$ Ser activos, no pasivos en el aprendizaje.

- Comprometerse en un enfoque de aprendizaje indagativo.

- Aceptar responsabilidad en su propio aprendizaje.

- Ser originales y creativos.

- Desarrollar habilidades de resolución de problemas, toma de decisiones y de evaluación.

- Desarrollar una visión amplia del mundo.

Una tarea adicional que le cabe al profesor es orientar al estudiante y proporcionarle las herramientas éticamente adecuadas para que pueda seleccionar adecuadamente, la información ofrecida por diferentes instituciones y personas, la que pudiera estar sesgada en función de los intereses institucionales.

En general, y desde el punto de vista del aprendizaje, el uso de variados recursos mejora en los alumnos la retención de la información debido a que emplean los diferentes sentidos en dicho proceso (tecnologías en las que se integran el color, el sonido, la imagen, el texto, a través de una interfaz que no sólo entretiene, sino que "facilita" el acceso y la búsqueda de información). En síntesis, en un modelo educativo basado en recursos, la escuela y el profesor dejan de ser fuentes de todo conocimiento, y el profesor pasa a ser un guía de alumnos para facilitarles el uso de recursos y herramientas que necesitan para explorar y elaborar nuevo conocimiento y destrezas, pasa a actuar como gestor de ambientes con recursos de aprendizaje y a acentuar su papel de orientador. El profesor podrá abordar temáticas de acuerdo a los intereses de sus alumnos, trabajar los contenidos al ritmo y estilo de aprendizaje 
del alumno en forma individual o grupal, y por último crear ambientes agradables que favorecen y facilitan el aprendizaje.

Es por ello que con este trabajo pretendemos desarrollar un modelo de autoaprendizaje con integración de las tecnologías de la información y el conocimiento, y los métodos de gestión del conocimiento que permita una comprensión y apropiación progresiva hasta socializar y producir el impacto que reclama la educación contemporánea del conocimiento.

\section{DESARROLLO}

Es indudable que la unidad básica de espacio educativo es el aula o la sala de clases y que la aparición de las TIC puede afectar directamente todo lo que ocurre dentro de ellas. Sin embargo, debemos precisar que los ambientes de aprendizaje enriquecidos con TIC no sustituyen las aulas tradicionales y que sólo tienen sentido en la medida que ocurran cambios en el modelo de enseñanza-aprendizaje a considerar, los roles de los participantes y de los medios. En síntesis, las TIC permiten diversificar los ambientes de aprendizaje. Según lo expuesto, los ambientes de aprendizaje diseñados a partir de un modelo educativo sustentado en recursos puede entenderse como "un marco o escenario destinado a promover el aprendizaje a partir de estrategias educativas enriquecidas con TIC que pretenden crear situaciones de aprendizaje que estimulen al máximo las potencialidades de los alumnos" o como "un conjunto articulado de condiciones que incluyen TIC destinadas al desarrollo de determinadas competencias en los estudiantes" (Soto, 2004). De acuerdo con estas definiciones, la flexibilidad del ambiente y el papel dinámico del profesor en una interacción intensa con sus alumnos, son elementos claves en los nuevos ambientes de aprendizaje.

Las herramientas que proporcionan los ambientes de aprendizaje informatizados ofrecen una serie de capacidades a los profesores, además de que favorecen un enfoque constructivista del aprendizaje, donde son los alumnos, en lugar de los profesores, quienes asumen la mayor parte del trabajo. Por tanto, en lugar de diseñar ambientes de aprendizaje basados en recursos de acuerdo a la figura de un profesor, debemos aprender a diseñarlos con el objetivo de aprovechar todas las posibilidades que estos ambientes informatizados nos ofrecen pensando en la construcción del conocimiento de cada uno de nuestros alumnos. 


\section{UN MODELO EDUCATIVO CENTRADO EN EL ESTUDIANTE PARA UNA PEDAGOGÍA DEL APRENDIZAJE}

Sin duda el objeto de interés de la pedagogía ha variado en el último siglo y medio. De establecerse en el siglo XIX como una teoría de la enseñanza, centrada en la didáctica experimental principalmente, a la luz de significativos desarrollos originados en la antropología, la psicología, la sociología, la biología y la misma pedagogía, entendida como campo de saber, ha trasladado, paulatinamente, su campo de interés al proceso de "aprendizaje", haciendo suyos los problemas relacionados con la interiorización del conocimiento y con las dimensiones, afectivas, sociales y contextuales que intervienen en ella. En particular, las corrientes constructivistas, y todas las vertientes en que ella se divide, han colocado especial interés en este tipo de aspectos, dando lugar a la exploración de un nuevo sistema de relaciones profesor/ alumno, que supera los enfoques memorísticos de la pedagogía tradicional y define nuevos roles, caracterizados por vínculos que en general se muestran más horizontales y comunicativos. El profesor dentro de esta concepción se entiende más como un "tutor" o un facilitador de los procesos de aprendizaje agenciados por los estudiantes.

De manera contemporánea, la discusión y comprensión en torno a los procesos de aprendizaje, se ha enriquecido gracias al impacto que han ejercido las tecnologías de la informática en el campo educativo, dinámica que se moviliza en un contexto determinado por una doble tensionalidad: de un lado la liberalización de la economía, que modifica profundamente las pautas de la formación para el trabajo y obliga a “aprender”, “desaprender” y “reaprender”, en ciclos cada vez más cortos, y del otro, una sobredemanda de los sistemas educativos convencionales a los que se les exige ampliar la cobertura con calidad y equidad. En este sentido, los cursos han de ser valorados, no tanto por su indiscutible carácter práctico, como por su potencialidad en términos de desencadenar procesos de autoaprendizaje, en los que se privilegia la reflexión y la crítica, y se coloca en escena la autogestión y la autonomía de los estudiantes, que de esta manera se constituyen en el centro del modelo educativo. A menudo la reflexión sobre los cursos se queda atrapada en el relieve que generan el "medio de comunicación" ocultando la dinámica pedagógica que ella origina, que es de suyo lo sustantivo del proceso.

Las nuevas exigencias del contexto, favorecidas por la dinámica de cambio del mundo actual y las expectativas del futuro que se construye, bajo la impronta de las Tecnologías de la Información y las Comunicaciones (TIC), surge la necesidad del redimensionamiento de las funciones y objetivos del proceso formativo, y por ende el replanteamiento de las categorías y leyes o regularidades didácticas que lo explican. 
Un análisis de las TIC en el ámbito de la educación y del resultado de su empleo ha permitido corroborar, que pese a sus logros y potencialidades éstas no han propiciado el efecto transformador esperado, debido a que de modo general la introducción de las mismas se ha efectuado de manera conjunta al modelo educativo existente. Por lo anterior, se considera que las reales potencialidades de las TIC sólo serán apreciables en la medida en que el nuevo modelo de enseñanza, en que éstas se inspiren, se formule en respuesta a las exigencias del mundo del futuro y en correspondencia con los nuevos principios y regularidades didácticas que caracterizan dicho proceso.

La universidad, como institución encargada de la transmisión de la cultura sistematizada por la humanidad, ya no puede proporcionar toda la información relevante, porque ésta es mucho más móvil y flexible que la propia institución, lo que sí puede es formar a los estudiantes para poder acceder y dar sentido a la información, proporcionándoles las capacidades de aprendizaje que les permitan una asimilación crítica de la misma. El sistema educativo universitario no puede formar específicamente para cada una de las necesidades, lo que sí puede hacer es formar a los futuros profesionales para que sean de por vida aprendices flexibles, eficaces y autónomos.

Coincidiendo con Homero (2003) nos encontramos en el proceso de formación a través de las TIC con tres dimensiones:

- Dimensión de extensibilidad.

- Dimensión de flexibilidad.

- Dimensión del cambio de roles.

Esas tres dimensiones tienen como base a la interactividad, la cual integra a las demás características de las Tecnologías de la Información.

En el Proceso de formación de los profesionales, la interactividad está determinada por la interacción individual del estudiante con el contenido y recursos de aprendizaje, y la interacción social del estudiante con el profesor, otros estudiantes, y otros actores del proceso, ya sea de forma simétrica donde éstos actúan en igualdad de condiciones, o complementaria, donde unos complementan a otros.

Según Homero (2003) la interactividad, trae aparejada las siguientes implicaciones: 
- El surgimiento de homólogos entre los cuales se establecen relaciones de colaboración, donde cada uno aporta su saber.

- El surgimiento de Comunidades, que son aquellas que están constituidas por conjuntos de homólogos.

- El trabajo colaborativo que se establece entre estudiantes, profesores y demás sujetos que pueden participar en el proceso, propicia el "crecimiento intelectual" tanto individual como colectivo.

Las posibilidades de las Tecnologías de la Información y las Comunicaciones en el proceso de formación de los profesionales favorecen la motivación de los contenidos y propicia la disposición e interés de los estudiantes para aprender, contribuyendo, a la vez, a una mejor comprensión de los contenidos.

Para nuestro modelo nos basamos en el modelo de gestión del conocimiento propuesto por Vizcaya, perfeccionado por Soto (2004) y a la vez contextualizado por los autores para lograr no sólo el acceso al conocimiento, sino una comprensión y apropiación progresiva hasta socializarlo y producir el impacto que reclama la educación contemporánea a partir de diagnosticar los problemas.

Para la confección del modelo se utiliza la siguiente leyenda para los gráficos:

- Las elipses para identificar las acciones que se realizan para la obtención del conocimiento.

- Los rectángulos identifican los procesos, medios y formas de obtener la información y el conocimiento.

- Las flechas identifican el ciclo e intercambio entre los procesos para lograr el aprendizaje. 


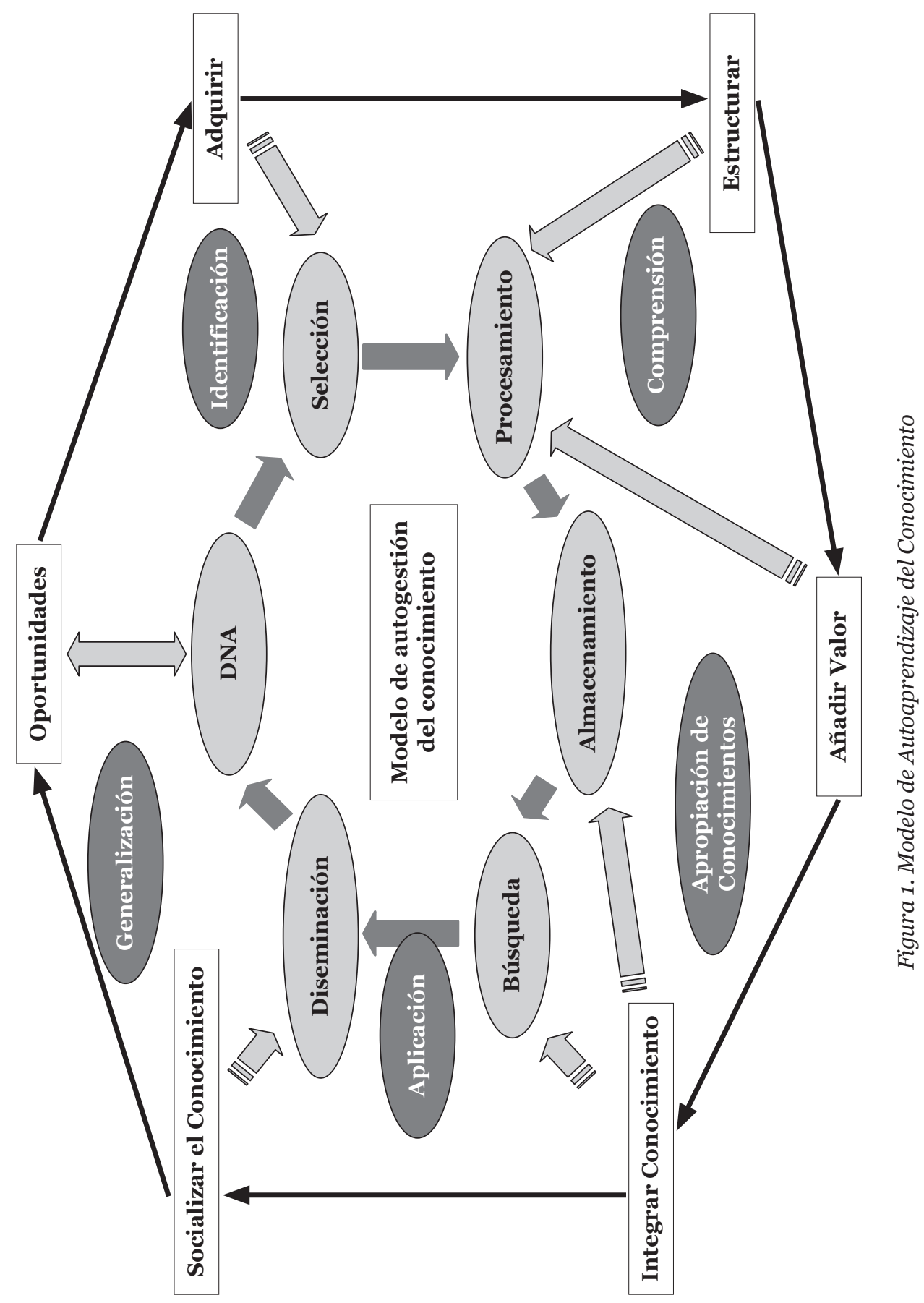


Analizando en nuestro modelo se hace viable la autogestión del conocimiento, a partir de determinar las necesidades de aprendizaje (DNA) de los estudiantes, vigilando la disponibilidad del entorno, posicionando nuestro saber en la materia, a partir de un diagnóstico estratégico sobre la preparación individual para enfrentar los desafíos de la carrera profesional, vida social universitaria y del barrio.

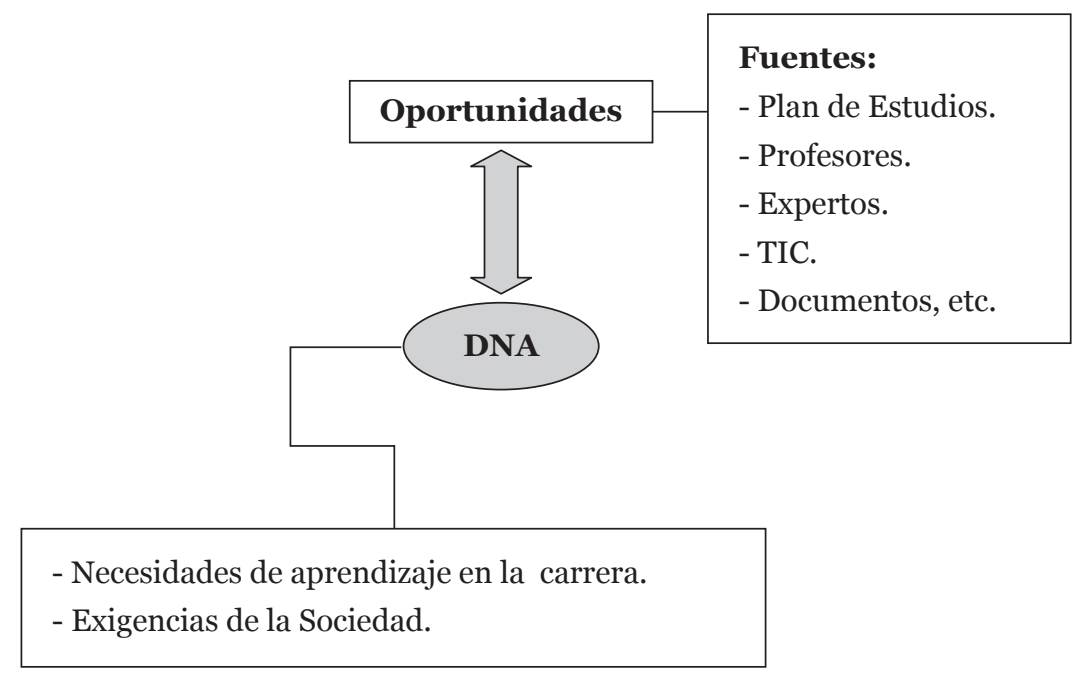

Figura 2. Articulación de la determinación de necesidades de aprendizaje y las oportunidades que se manifiestan en el entorno

Una vez logrado el acceso a las diferentes fuentes de información se adquieren estas (artículos, informes e informaciones, entrevistas con investigadores, etc.). Esta información se selecciona (ordenar, clasificar, etc.) en correspondencia con las necesidades de aprendizajes antes definidas. En este momento se identifican los conocimientos necesarios para dar respuesta a los retos del saber, impuestos por el diagnóstico inicial. 
- Clases.

- Radio y TV.

- Intercambios con otros.

- TIC.

- Lectura de Materiales, etc.

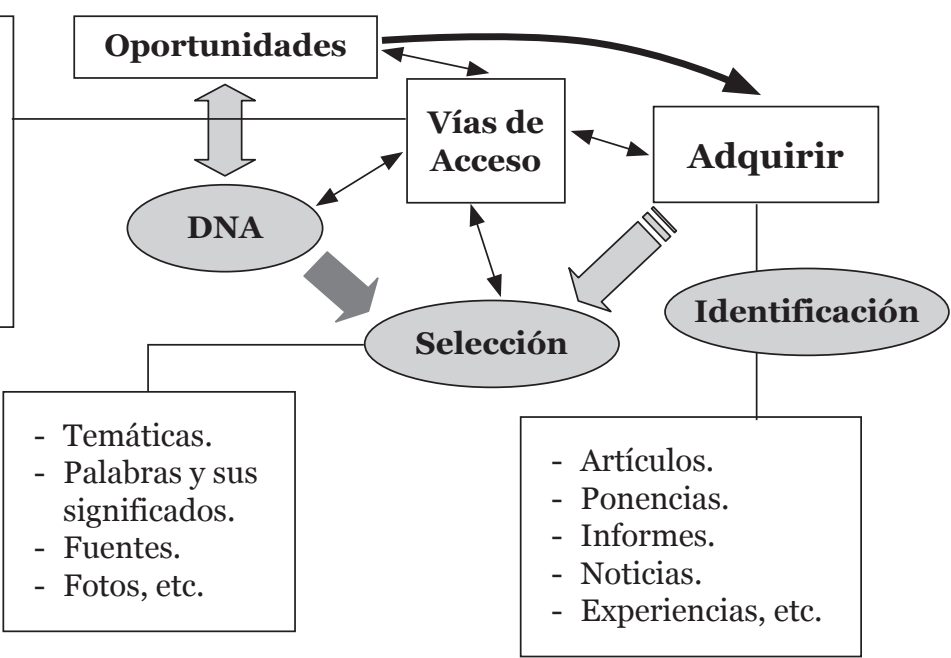

Figura 3. Vías de acceso para adquirir y seleccionar la información demandada

La estructuración y procesamiento de la información contribuye a la organización de la información disponible para garantizar entre otras cosas un fácil y ágil acceso. A partir de entonces realmente se ha iniciado la comprensión de los contenidos que responden a las necesidades de aprendizaje.

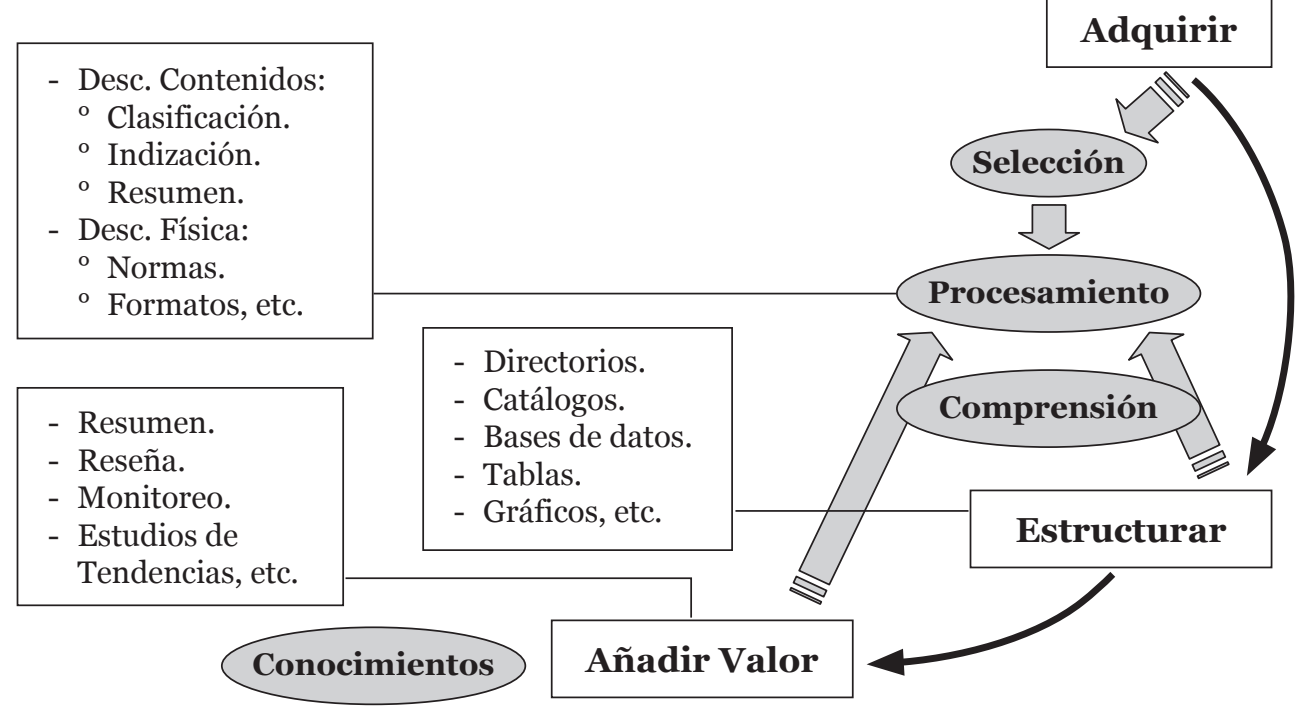

Figura 4. Estructuración, procesamiento y valor agregado de la información 
El valor añadido se alcanza elaborando resúmenes, reseñas, bases de datos, etc., a partir de entonces declaramos entonces que estamos adquiriendo conocimientos demostrando así la apropiación de los mismos por parte de los estudiantes.

El almacenamiento se hace integrando el conocimiento, creando sistemas de contenidos para facilitar con precisión las búsquedas.

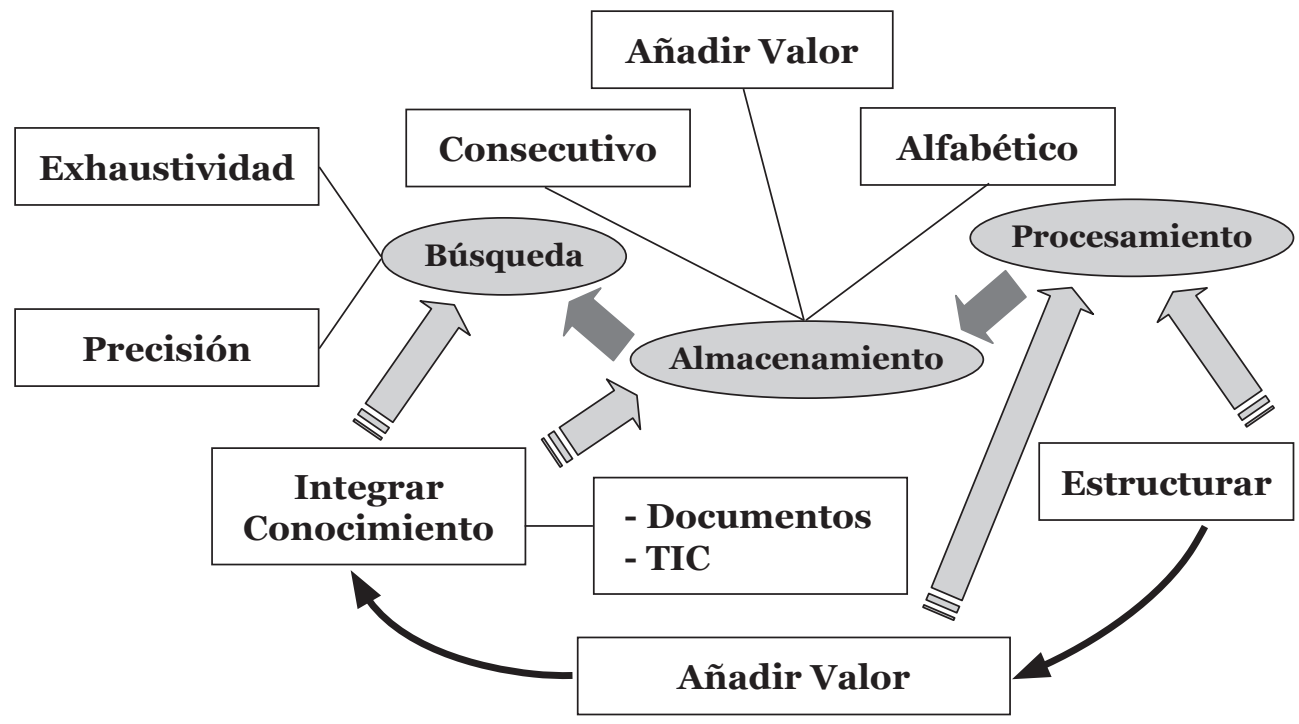

Figura 5. Almacenamiento y búsqueda de la información y el conocimiento

La diseminación y socialización de los conocimientos se realiza cuando el estudiante presenta los resultados de alguna forma en eventos, seminarios y talleres demostrando de esa manera lo aprendido. 


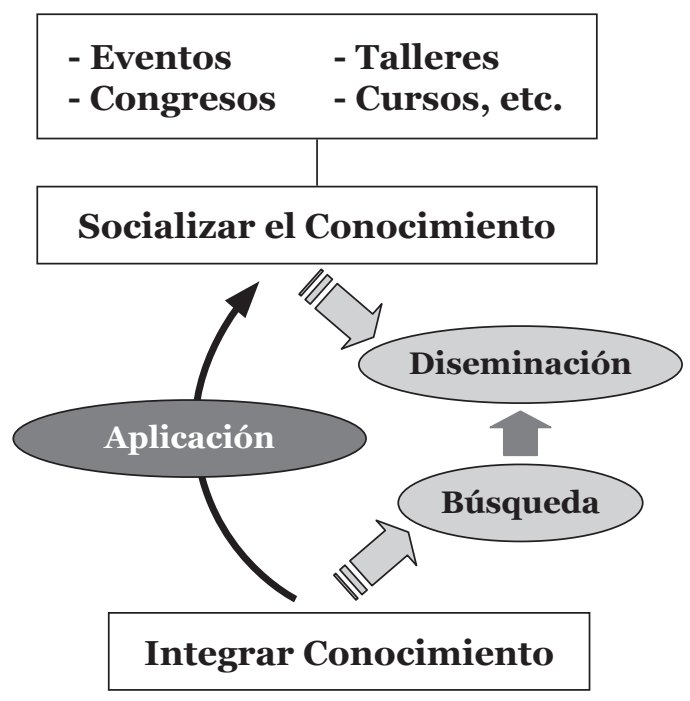

Figura 6. Diseminación y socialización del conocimiento

Todo esto ha traído consigo la aplicación y generalización del trabajo del estudiante completando de esa forma el ciclo de autoaprendizaje.

La calidad de la educación en este modelo, descansa en los siguientes pilares:

- Fuerza de trabajo intelectual congruente con la información de calidad.

- Tiempo invertido al aprendizaje (número de horas dedicadas.

- Información humanística, científica y tecnológica de la mejor calidad.

- Habilidades en la autogestión del conocimiento.

- Productos intelectuales generados.

- La alerta y el uso eficiente de la tecnología.

- Lenguaje profesional incorporado.

- Socialización asesor-alumno-TIC.

- Valoración sistemática.

- Soporte tecnológico. 


\section{CONCLUSIONES}

Los profesores necesitamos ser guías en el proceso de aprendizaje, más que funcionar como los únicos expertos. Podemos enseñar a los estudiantes cómo aprender a buscar, recolectar, analizar y comunicar información. Esta habilidad de descubrir, usar y presentar información efectivamente será una de las claves del éxito en el mercado de trabajo actual y futuro. De lo presentado y a modo de síntesis puede decirse que las nuevas coordenadas espacio-temporales configuradas por las TIC, y las experiencias basadas en dichas concepciones, no sólo afectan a la comunicación educativa como tal sino a todo el sistema y proceso educativo. Además podemos concluir en cuanto al modelo:

- El modelo de gestión del conocimiento empleado en el autoaprendizaje es efectivo para desarrollar la educación.

- El modelo empleado permite además transitar desde la determinación de las necesidades de aprendizaje hasta la aplicación y socialización de los conocimientos adquiridos.

- El modelo de gestión del conocimiento usado permite continuar la educación en la medida que surjan otras necesidades de aprendizaje.

\section{REFERENCIAS BIBLIOGRÁFICAS}

Alvero, F. (1978). Diccionario Manual de la Lengua Española. La Habana: Ed. Pueblo y Educación (932).

Fernández Aedo, R. (2007). La virtualización en la universalización de la enseñanza. Journal of Technology Management \& Innovation. [en línea] Disponible en: http://www.jotmi.org [consulta 2007, 11 de mayo].

Gispert,C.(1999).Enciclopediaautodidáctica interactiva OCEANO. Barcelona, España: Editorial OCEANO.T 6 y 5 .
Homero Fuente, L. (2003). Modelo Informático de universalización. Cuba: Editorial Santiago.

Soto Balbon, M. A. (2004). Propuesta de aplicación de un modelo de Gestión del conocimiento para las entidades del CITMA. Habana: Dirección de Tecnologías de Información y Gestión del Conocimiento (TIGEC). 


\section{PERFIL ACADÉMICO Y PROFESIONAL DE LOS AUTORES}

Raúl Rubén Fernández Aedo. Licenciado en Matemáticas, Master en Computación Aplicada, Doctor en Ciencias en Aportaciones Humanistas y Sociales por la Universidad de Granada, España y Doctor en Ciencias Pedagógicas por Cuba. Es profesor auxiliar de la Universidad de Ciego de Ávila, Cuba. Posee múltiples participaciones en eventos Nacionales e Internacionales así como publicaciones en revistas. Sus principales líneas investigativas están encaminadas al desarrollo de software educativos, gestión del conocimiento y educación a distancia.

E-mail: $\underline{\text { aedo@informatica.unica.cu }}$

Elme Carballo Ramos. Ingeniero Mecanizador graduado en la Universidad de Minks, Doctor en Ciencias Técnicas, especialista en Gestión del Conocimiento. Es profesor titular de la Universidad de Ciego de Avila, Cuba. Ha participado en múltiples eventos nacionales e internacionales y posee varias publicaciones en revistas especializadas. Coordina doctorados internacionales en Gestión del Conocimiento y Pedagogía.

Martín Delavaut Romero. Licenciado en Informática, colabora activamente con la Universidad de Comaguen, Argentina. Posee varias participaciones en eventos internacionales y publicaciones. Es miembro de un grupo de desarrollo de plataformas y portales educativos.

DIRECCIÓN DE LOS AUTORES:

Raúl Rubén Fernández Aedo

Avenida Tarafa \#4 entre Dimas Daniel y

Coronel Cervantes.

Morón. Ciego de Avila. Cuba.

Cod. Postal: 67210

Martín Delavaut Romero

Avenida Libertador \#27128.

Comahue. Argentina.

Fecha de recepción del artículo: 20/11/07

Fecha de aceptación del artículo: 13/03/08 